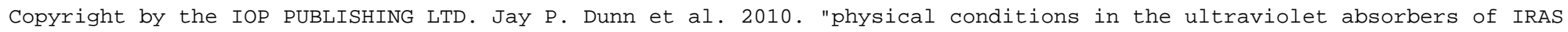
F22456-5125," ApJ 713900 doi:10.1088/0004-637X/713/2/900

The Astrophysical Journal, 713:900-905, 2010 April 20

\title{
PHYSICAL CONDITIONS IN THE ULTRAVIOLET ABSORBERS OF IRAS F22456-5125*
}

\author{
Jay P. Dunn ${ }^{1}$, D. Michael Crenshaw ${ }^{2}$, S. B. Kraemer ${ }^{3}$, and M. L. Trippe ${ }^{2}$ \\ ${ }^{1}$ Department of Physics, Virginia Tech, Blacksburg, VA 24061, USA; jdunn77@ vt.edu \\ ${ }^{2}$ Department of Physics and Astronomy, Georgia State University, Atlanta, GA 30303, USA; crenshaw@ @ chara.gsu.edu, trippe@chara.gsu.edu \\ ${ }^{3}$ Institute for Astrophysics and Computational Sciences, Department of Physics, The Catholic University of America, Washington, DC 20064, USA; \\ kraemer@yancey.gsfc.nasa.gov \\ Received 2009 July 24; accepted 2010 February 16; published 2010 March 29
}

\begin{abstract}
We present the ultraviolet (UV) and X-ray spectra observed with the Far Ultraviolet Spectroscopic Explorer (FUSE) and the XMM-Newton satellite, respectively, of the low- $z$ Seyfert 1 galaxy IRAS F22456-5125. This object shows absorption from five distinct, narrow kinematic components that span a significant range in velocity $(\sim 0$ to $-700 \mathrm{~km}$ $\mathrm{s}^{-1}$ ) and ionization (Lyman series, $\mathrm{C}$ III, N III, and $\mathrm{O}$ VI). We also show that three of the five kinematic components in these lines appear to be saturated in $\operatorname{Ly} \beta \lambda 1026$ and that all five components show evidence of saturation in the $\mathrm{O}$ VI doublet lines $\lambda \lambda 1032$, 1038. Further, all five components show evidence for partial covering due to the absorption seen in the $\mathrm{O}$ vi doublet. This object is peculiar because it shows no evidence for corresponding X-ray absorption to the UV absorption in the X-ray spectrum, which violates the 1:1 correlation known for low- $z$ active galactic nuclei (AGNs). We perform photoionization modeling of the UV absorption lines and predict that the O VII column density should be small, which would produce little to no absorption in agreement with the X-ray observation. We also examine the UV variability of the continuum flux for this object (an increase of a factor of 6). As the absorption components lack variability, we find a lower limit of $\sim 20 \mathrm{kpc}$ for the distance for the absorbers from the central AGN.
\end{abstract}

Key words: galaxies: Seyfert - ultraviolet: galaxies

\section{INTRODUCTION}

It has become well known that most massive galaxies host a supermassive black hole in their core (e.g., Kormendy \& Richstone 1995; Gebhardt et al. 2003). Active galactic nuclei (AGNs) are galaxies where the black hole is surrounded by an accretion disk, which provides immense luminosity. Seyfert galaxies are moderate-luminosity AGNs $\left(L_{\text {Bol }} \approx 43-45 \mathrm{erg} \mathrm{s}^{-1}\right)$ that were first classified by Seyfert (1943), and were later divided into two categories (Seyfert 1 and Seyfert 2) by Khachikian \& Weedman (1974) based on spectroscopic observations of their emission lines. Seyfert 1 galaxies contain broad emission lines (BELs; FWHM $\approx 1000-5000 \mathrm{~km} \mathrm{~s}^{-1}$ ) of permitted transitions covering a large range in ionization. By contrast, Seyfert 2 galaxies only exhibit narrow emission lines (NEL; FWHM $\approx 500-800 \mathrm{~km} \mathrm{~s}^{-1}$ ), which arise from both forbidden and permitted transitions. This was later explained in a unified model (Antonucci \& Miller 1985; Urry \& Padovani 1995), where a putative torus obscures the BEL region in Seyfert 2 galaxies.

It is found in many cases that the BELs of Seyfert 1 galaxies observed in the ultraviolet (UV) have blueshifted absorption features that are associated with outflowing gas from the AGN. Crenshaw et al. (1999) showed in a survey of Seyfert galaxies, using data from the Faint Object Spectrograph (FOS) and Goddard High Resolution Spectrograph (GHRS) previously on board the Hubble Space Telescope (HST), that UV absorbers in C IV $\lambda \lambda 1048,1551$ are relatively common $(\sim 60 \%)$. Another survey by Dunn et al. (2007) of Far Ultraviolet Spectroscopic Explorer (FUSE) archival data showed that these outflows are also common in the $\mathrm{O}$ VI doublet $\lambda \lambda 1032,1038$ and $\operatorname{Ly} \beta \lambda 1026$ lines $(\sim 50 \%)$ with a global covering factor $\left(\left\langle C_{g}\right\rangle\right) \approx 0.4$ and that the O vi absorption features frequently vary $(\sim 40 \%$ of the objects) in low- $z$ AGNs.

\footnotetext{
* Based on observations made with the NASA-CNES-CSA Far Ultraviolet Spectroscopic Explorer. FUSE is operated for NASA by the Johns Hopkins University under NASA contract NAS5-32985.
}

In our survey, we discovered the intrinsic UV absorption lines in the Seyfert 1 galaxy IRAS F22456-5125 (RE J2248-511, $z=0.1016 \pm 0.0001)$. The luminosity of this object is $\log L_{\mathrm{Bol}}$ $\left(\mathrm{erg} \mathrm{s}^{-1}\right)=45.53$ (Dunn et al. 2008). Given the redshift and luminosity of this object, it could be considered a low- $z$ quasar. However, the spectrum of IRAS F22456-5125 is more similar to that of Seyfert 1 galaxies with strong narrow emission lines. We also found that this object was unique amongst the other objects with $\mathrm{O}$ VI absorption as it showed five relatively narrow and separable absorption features, as opposed to blended and saturated absorption troughs found in other objects, situated in a velocity range that spans $700 \mathrm{~km} \mathrm{~s}^{-1}$. Due to its singular nature, we selected this object to evaluate in more detail.

Currently, there are several UV observations of IRAS F22456-5125. In the UV, the International Ultraviolet Explorer (IUE) observed this object five times and FUSE observed it three times. The continuum flux history of IRAS F22456-5125 is given by the IUE data, as shown by the online AGN Light Curve Database, ${ }^{4}$ which shows that IRAS F22456-5125 was in a low-flux state during the observations taken with FUSE. In the optical regime we have obtained two spectra from CTIO (published in Dunn et al. 2008) and two spectroscopic observations were taken with $2.2 \mathrm{~m}$ and $2.1 \mathrm{~m}$ telescopes at La Silla, Chile, and McDonald Observatory of the University of Texas at Austin, respectively (Mason et al. 1995; Grupe et al. 1998). These spectra were published in a figure later in Breeveld et al. (2001) and appeared to show that the continuum slope varied drastically over the span of a year.

IRAS F22456-5125 has also been observed several times in the extreme UV and X-ray regimes. It was first detected in a ROSAT observation by Pounds et al. (1993). They noted that this object belongs to uncommon type of Seyfert galaxies with a steep spectral component at energies $<1 \mathrm{keV}$. It was later observed by the Advanced Satellite for Cosmology and

\footnotetext{
4 http://www.chara.gsu.edu/PEGA/IUE/ (Dunn et al. 2006).
} 


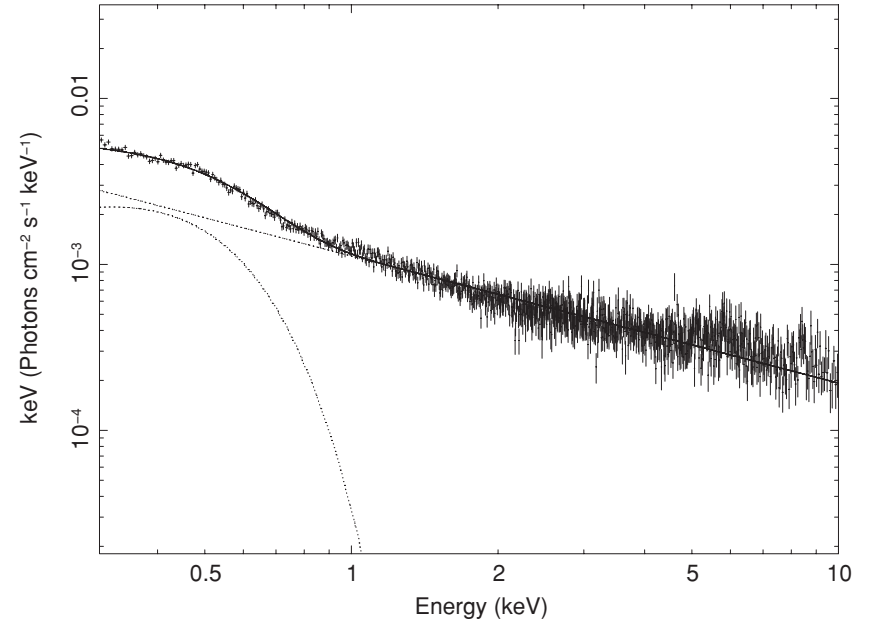

Figure 1. Data taken by the XMM-Newton satellite (crosses) and our fit (solid line) for IRAS F22456-5125. We show in dotted lines the power-law fit to the hard X-ray data $(\Gamma=1.77)$ and a soft Gaussian X-ray excess at energies less than $1 \mathrm{keV}$. We note the lack of evidence for an X-ray warm absorption edge in these data.

Astrophysics (ASCA). Both the ROSAT and ASCA data points are published by Breeveld et al. (2001). Finally, IRAS F552456-5125 has three previously unpublished XMM-Newton observations.

We begin in Section 2 with an analysis of the X-ray observations and discuss the shape of the ionizing spectral energy distribution (SED). In Section 3, we examine the individual FUSE spectra and measure several parameters (e.g., ionic column densities). Section 4 provides a brief look at the time variability of the system, and in Section 5 we outline our conclusions.

\section{X-RAY ANALYSIS}

Breeveld et al. (2001) approximated the SED from the available observations in the X-ray, UV, and optical. They noted that IRAS F22456-5125 showed two interesting features. The first was that the X-ray observations showed evidence for an ultrasoft X-ray excess, and the second was that the optical spectra drastically changed slopes over the span of a year. Their power-law fits $\left(F_{v} \propto v^{\alpha}\right)$ for the X-ray were $\alpha=-1.3$ and $\alpha=-0.8$ for the soft $(0.3-2 \mathrm{keV})$ and hard X-ray (2-10 keV), respectively, and a very high value of $\alpha=-3.1$ for the ultrasoft X-ray excess $(0.1-0.3 \mathrm{keV})$. They claim that this excess, however, appears to be variable over time along with the slope in the optical regime. The optical slopes vary between $\alpha=-1.3$ in the 1992 observation from Grupe et al. (1998) and $\alpha=+0.8$ in the 1991 observation from Mason et al. (1995).

In order to better evaluate the X-ray spectrum of IRAS F22456-5125, we have retrieved the archived XMM-Newton X-ray observations taken with the European Photon Imaging Camera (EPIC) pn detector. The observation (ID 0510380101) was taken over the dates of 2007 May 15 and 16 at 10:37, with an exposure time of $4.506 \mathrm{ks}$. We reduce the data using the standard EPCHAIN processing script included in version 7.1.0 of the XMM-Newton Science Analysis System (XMMSAS) software. The data were filtered to exclude non-X-ray events; only events with event patterns in the range $0-4$ (single and double pixel events) were kept. The source spectrum was extracted from a $32^{\prime \prime}$-radius circle, the size of the X-ray point source on the PN chip. The background spectrum was extracted from another $32^{\prime \prime}$ circle on the same chip as the source, but from

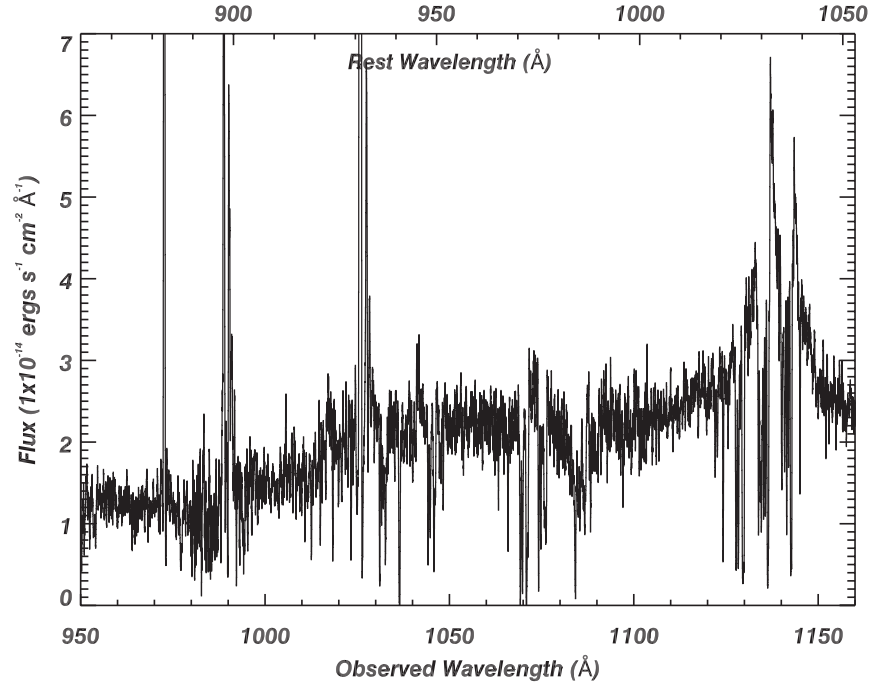

Figure 2. Average far-UV spectrum of IRAS F22456-5125 based on three observations with FUSE. These data have been boxcar smoothed by 7 pixels. The rest-frame wavelength is indicated across the top axis, and the $\mathrm{O}$ VI doublet $\lambda \lambda 1032,1038$ broad emission can be clearly seen as well as the narrow absorption features.

an area free of any background sources. We generate response matrices and ancillary response matrices using the XMM-SAS tasks rmfgen and arfgen. The resulting background-subtracted spectrum is shown in Figure 1. The XMM-Newton spectrum of IRAS $22456-5125$ is well fit $\left(\chi^{2}=1234.94\right.$ for 953 degrees of freedom) by a power law with photon index $\Gamma=1.77$ and a soft excess below $1 \mathrm{keV}$ (modeled simplistically in our spectrum with a Gaussian).

We examine and find no evidence for an X-ray warm absorption edge in these data, as shown in Figure 2. The lack of absorption, combined with the power laws prescribed by Breeveld et al (2001), leads us to believe that IRAS F22456-5125 is devoid of strong X-ray absorbers. These XMMNewton data were taken approximately two years after the data from FUSE and when combined with the Breeveld et al. study show that the soft X-ray excess has existed for several years. A consequence of this long-standing X-ray excess, which spans a range that includes the FUSE observations, is that the lack of corresponding X-ray absorption must be due to a paucity of $\mathrm{X}$-ray absorbing gas in the cloud.

The "soft excess" also gives information about the AGN itself. Frequently, low-z AGNs (e.g., Reeves \& Turner 2000; Piconcelli et al. 2005) show a soft X-ray excess and it has been shown that the soft X-ray spectral slope presence is more common in high Eddington ratio $\left(L / L_{\text {Edd }} \approx 1\right.$; Grupe 2004; Czerny et al. 2003) objects such as narrow-line Seyfert 1 galaxies (NLS1). However, despite this excess, Dunn et al. (2008) have shown with optical spectra that IRAS F22456-5125 is not a high accretion rate object with an Eddington ratio of only $L / L_{\text {Edd }} \approx 0.16$ and furthermore showed that IRAS F22456-5125 is not an NLS1 galaxy (i.e., $\operatorname{FWHM}(\mathrm{H} \beta) \approx 3300 \mathrm{~km} \mathrm{~s}^{-1}$ ).

\section{FUV ANALYSIS}

We obtained the archived FUSE spectra from the Multimission Archive at Space Telescope (MAST) presented in Table 1. We processed these data with the CalFUSE reduction package (v3.2) in time-tag mode (TTAG; Dixon et al. 2002, see Dunn et al. 2007 for full details). The output data consist of 8 spectra per observation for each grating and detector, which we weighted by exposure time per segment of the detector, and then 
Table 1

FUV Observations

\begin{tabular}{lccc}
\hline \hline \multicolumn{1}{c}{ Date } & Julian Date $^{\mathrm{a}}$ & Observation ID & Exposure Time (s) \\
\hline 2002 Sep 24 09:40:18 & 52541.902 & Z9073901 & 5721 \\
2002 Sep 24 22:56:34 & 52542.457 & Z9073902 & 31836 \\
2004 Jul 7 21:59:25 & 53194.629 & E8481401 & 3403 \\
\hline
\end{tabular}

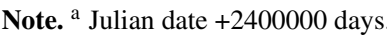

co-added across the segments and scaled to match the flux in the segment that was used for primary alignment of the object (the LiF 1a segment). We show the full FUSE spectrum in Figure 2.

As we have stated, IRAS F22456-5125 shows an interesting assortment of intrinsic absorption lines in the FUV. Currently, there are no observations of IRAS F22456-5125 with sufficient resolution to examine the $\mathrm{C} I \mathrm{~V}, \mathrm{~N} \mathrm{v}$, and $\mathrm{Si}$ IV doublets for absorption. The five kinematic components detected in the FUSE data span a velocity range of $700 \mathrm{~km} \mathrm{~s}^{-1}$, which we number from highest velocity to lowest $1-5$ (see Table 2 ). Three of these five components (2, 3, and 5) are strong in $\operatorname{Ly} \beta \lambda 1025.7$, $\mathrm{C}$ III $\lambda$ 977.0, $\operatorname{Ly} \gamma \lambda$ 972.5, $\operatorname{Ly} \delta \lambda 949.7, \mathrm{Ly} \eta \lambda 937.8$, and possibly $\mathrm{N}$ III $\lambda 989.7$ and Ly $6 \lambda 930.8$. We show the first four of these and the $\mathrm{O}$ vi doublet in Figure 3, indicated with dotted lines for the five components. We note that components 1 and 4 are much weaker in the higher order Lyman series lines but comparable to components 2 and 3 in the $\mathrm{O}$ VI lines.

In the three FUSE spectra, we find that the five absorption lines in IRAS F22456-5125 remain constant in FWHM and equivalent width for the $\mathrm{O}$ VI doublet, $\mathrm{Ly} \beta, \mathrm{Ly} \gamma$, and $\mathrm{C}$ III (which have only moderate signal-to-noise ratio $(\mathrm{S} / \mathrm{N}))$ over a time range of $\sim 450$ days, and the UV continuum flux increases by a factor of 6 . We co-add all three available spectra weighted by exposure time for better $\mathrm{S} / \mathrm{N}$, due to the lack of variability in the absorption components, and present the spectrum sections with the measurable lines in Figure 3. With so many lines present and a good range of ionization, IRAS F22456-5125 is an excellent candidate for photoionization modeling of the absorption.

To begin, we measure the residual intensity $\left(I_{r}\right)$, normalized by nearby continuum points, for each of the $\mathrm{O}$ vI lines and $\mathrm{Ly} \beta$ and calculate the line-of-sight (LOS) covering factors $\left(C_{f}\right)$ for the components using the doublet method (Hamann et al. 1997):

$$
C_{f}=\frac{I_{1}^{2}-2 I_{1}+1}{I_{2}-2 I_{1}+1},
$$

where $I_{1}$ and $I_{2}$ are the normalized intensities for the blue and red lines in the doublet, respectively ( $\lambda 1032$ and $\lambda 1038$ for $\mathrm{O} \mathrm{VI}$ ). In many cases, the LOS covering factor can be a function of velocity. To perform this analysis, the data must be both high resolution $(R \gtrsim 10,000)$ and high $\mathrm{S} / \mathrm{N}(\gtrsim 20)$ in the continuum. While FUSE provides sufficient resolution, the $\mathrm{S} / \mathrm{N}$ is only 7
(Dunn et al. 2007). Thus, we use the maximum trough depths to determine the LOS covering factor.

To calculate the LOS covering factor of the Lyman lines (as covering factor is not necessarily the same from ion to ion), we utilize $\operatorname{Ly} \beta$ and $\operatorname{Ly} \gamma$. These are not a doublet, and Equation (2) cannot be used to solve for the LOS covering. Instead, we can calculate the LOS covering and optical depth by simultaneously solving a system of equations for normalized intensity of two troughs with partial LOS covering:

$$
I=1-C_{f}+C_{f} e^{-r \tau},
$$

where $I$ is the normalized intensity and $r$ is the ratio of oscillator strengths and wavelengths ( $\sim 2.08$ for the ratio of $\operatorname{Ly} \beta$ to $\operatorname{Ly} \gamma)$. We present these LOS covering factor solutions in Table 2.

For a saturated line, a good approximation for the covering factor should be $1-I_{r}$. Comparing the LOS covering factors and the residual intensities of $\operatorname{Ly} \beta$, we find that components 2,3 , and 5 are saturated given the flux measurement errors. Components 1 and 4 are the weaker components in Ly $\beta$, and do not appear to be saturated. We measure $1-I_{r}$ for $\mathrm{O}$ vi $\lambda 1038$ (O vir), and find that all five lines are comparable and are therefore saturated. We note that the $1-I_{r}$ measurements of $\mathrm{O}$ VI $r$ should not exceed the values we derive from the $\mathrm{O}$ vi doublet as this is the maximum depth for a saturated line.

Provided with the covering factors from Table 2, we are able to convert from normalized intensity to optical depth. Hamann et al. (1997) shows that the optical depth for a line from an absorber that does not completely cover the source is given by

$$
\tau=\ln \frac{C_{f}}{I_{r}+C_{f}-1},
$$

where $\tau$ is evaluated at each radial velocity $\left(v_{r}\right)$. This equation combined with (Savage \& Sembach 1991)

$$
N=\left(\frac{m_{e} c}{\pi e^{2} f \lambda}\right) \int \tau\left(v_{r}\right) d v_{r},
$$

where $f$ is the oscillator strength of the line, gives the column density for the line in question.

Because components 2, 3, and 5 are saturated, $\tau$ approaches infinity for $\operatorname{Ly} \beta$. Thus, we do not include $\operatorname{Ly} \beta$ for the calculation of the $\mathrm{H}$ I column density in these components. Ly $\gamma$ shows the same trait for component 3. Components 2 and 5, however, are measurable in $\operatorname{Ly} \gamma$ and $\operatorname{Ly} \delta$, while $\operatorname{Ly} \delta$ and $\operatorname{Ly} \eta$ provided good estimates for component 3 . For each component, we find at least two lines, for consistency, that are measurable and allow us to calculate an $\mathrm{H}$ I column density, shown in Table 3.

The troughs for component 2 are contaminated in both $\mathrm{O}$ VI doublet lines. We see that an Fe II interstellar medium (ISM) line is blended with $\mathrm{O}$ VI $\lambda 1032$. The $\mathrm{O}$ VI $\lambda 1038$ component

Table 2

Covering Factors

\begin{tabular}{lcccccccc}
\hline \hline Comp & $\begin{array}{c}\text { Velocity } \\
\left(\mathrm{km} \mathrm{s}^{-1}\right)\end{array}$ & $\begin{array}{c}\text { FWHM } \\
\left(\mathrm{km} \mathrm{s}^{-1}\right)\end{array}$ & $\begin{array}{c}C_{f} \\
(\mathrm{Ly} \beta)\end{array}$ & $\begin{array}{c}C_{f} \\
(\mathrm{O} \text { VI })\end{array}$ & $\begin{array}{c}1-I_{r} \\
(\mathrm{Ly} \beta)\end{array}$ & $\begin{array}{c}\sigma_{I} \\
(\mathrm{Ly} \beta)\end{array}$ & $\begin{array}{c}1-I_{r} \\
(\mathrm{O} \text { VIr })\end{array}$ & $\begin{array}{c}\sigma_{I} \\
(\mathrm{O} \text { VIr })\end{array}$ \\
\hline 1 & -801 & 58 & $1.0^{\mathrm{a}}$ & 0.72 & 0.11 & 0.13 & 0.69 & 0.11 \\
2 & -608 & 112 & 0.84 & 0.69 & 0.92 & 0.13 & 0.64 & 0.11 \\
3 & -442 & 97 & 0.86 & 0.84 & 0.90 & 0.13 & 0.83 & 0.10 \\
4 & -320 & 44 & 0.70 & 0.66 & 0.35 & 0.12 & 0.62 & 0.10 \\
5 & -130 & 183 & 0.93 & 0.95 & 1.00 & 0.12 & 0.95 & 0.10 \\
\hline
\end{tabular}

Note. ${ }^{a}$ Used apparent optical depth for the column density limit. 


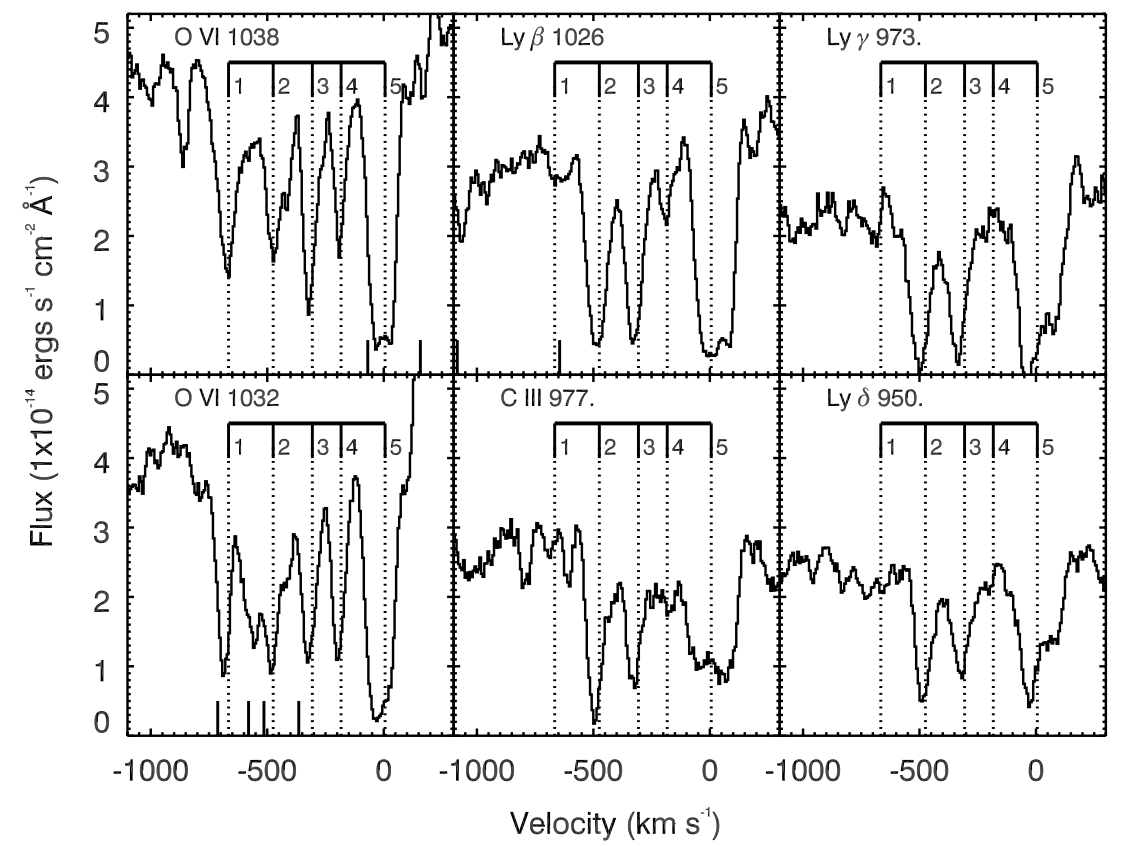

Figure 3. Spectral absorption regions in order of decreasing wavelength from top to bottom and left to right. Each of the dotted lines represents the average velocity for the five kinematic components averaged over the three available FUSE observations (see Dunn et al. 2008) and labeled accordingly. The solid black tick marks at the bottom of the plots indicate common ISM transitions. With the exception of the O vI $\lambda 1032$ panel, the lines are from Fe II. However, these lines cannot contribute much contamination as the strongest line (Fe II $\lambda 1143$ ) is not clearly seen. The only lines not from Fe II are the three redward lines in the $\mathrm{O}$ VI $\lambda 1032$ panel, which come from N I. These data have been boxcar smoothed by 7 pixels.

Table 3

Column Densities ${ }^{\mathrm{a}}$

\begin{tabular}{lrrrrrrrr}
\hline \hline Components & $N_{\mathrm{HI}}$ & $\sigma_{\mathrm{HI}}$ & $N_{\mathrm{OVI}}$ & $\sigma_{\mathrm{OVI}}$ & $N_{\mathrm{N}_{\text {III }}}$ & $\sigma_{\mathrm{N}_{\text {III }}}$ & $N_{\mathrm{C} \text { III }}$ & $\sigma_{\mathrm{C}_{\text {III }}}$ \\
\hline 1 & $<9$ & $\ldots$ & 8.9 & 2.8 & $<0.2$ & $\ldots$ & $<0.03$ & $\ldots$ \\
2 & 44.1 & 6.6 & $>8.8$ & $\ldots$ & 6.4 & 1.7 & 1.8 & 0.4 \\
3 & 42.3 & 7.9 & $>8.7$ & $\ldots$ & $<0.6$ & $\ldots$ & 1.3 & 0.2 \\
4 & 5.5 & 1.8 & 5.2 & 2.3 & $<0.1$ & $\ldots$ & $<0.5$ & $\ldots$ \\
5 & 60.1 & 12 & $>14$ & $\ldots$ & $<0.6$ & $\ldots$ & 1.3 & 0.8 \\
\hline
\end{tabular}

Note. ${ }^{\text {a }}$ Ionic column densities are given in units of $\times 10^{14} \mathrm{~cm}^{-2}$.

2 trough has an unidentified trough partially blended with the outflow kinematic component. Both of these can be seen in Figure 3. We attempt to exclude these features and measure only the absorption line related to the outflow. We provide the estimates of column densities for $\mathrm{O}$ VI, C III, N III, and H I in Table 3. Our error estimates are derived from the standard deviation of multiple lines of the same ion. This does not apply to C III as there is only one line for measurement. Therefore, we propagate the dominant source of the error (the error in flux) through Equations (3) and (4) to find the error in column density for components 2,3 , and 5 for which we have measurements. With the exception of component 2 , the $\mathrm{N}$ III lines are very weak, if present, in the spectrum and thus we provide only an upper limit on them. Furthermore, there is an N II $\lambda 1084$ ISM line that lies at the same wavelength of component 2 in $\mathrm{N}$ II, which leads to an overestimate for the column density. Components 2 , 3 , and 5 are only lower limit measurements for $\mathrm{O}$ vI, because as we have shown $1-I_{r}$ is very close to the estimated covering factor, and we therefore assume that the lines are saturated.

\section{PHOTOIONIZATION MODELS}

The photoionization models used for this study are generated using the 07.02.01 version of Cloudy (Ferland et al. 1998). The models are characterized by an ionization parameter, $U, 5$ and hydrogen column density, $N_{\mathrm{H}}=N_{\mathrm{H}^{0}}+N_{\mathrm{H}^{+}}$(in units of $\mathrm{cm}^{-2}$ ). We assume roughly solar elemental abundances (e.g., Grevesse \& Anders 1989) and that the absorbing gas is free of cosmic dust. The logs of the elemental abundances, relative to $\mathrm{H}$ by number, are as follows: He: $-1.00, \mathrm{C}:-3.47, \mathrm{~N}:-3.92, \mathrm{O}:-3.17, \mathrm{Ne}$ : -3.96, Na: -5.69 , Mg: $-4.48, \mathrm{Al}:-5.53, \mathrm{Si}:-4.51, \mathrm{P}:-6.43$, S: -4.82 , Ar: -5.40 , Ca: -5.64 , Fe: -4.40 , and Ni: -5.75 . Because the predicted ionic column densities are not sensitive to density for $n_{\mathrm{H}}<10^{11} \mathrm{~cm}^{-2}$, and we have no solid constraints on the radial distances of the absorbers, we assumed $n_{\mathrm{H}}=10^{5}$ $\mathrm{cm}^{-3}$.

We base our model intrinsic SED on the $1020 \AA$ flux observed with FUSE and the X-ray fluxes from Breeveld et al. (2001), as detailed in Section 2. We assume a broken power law of the form $L_{v} \propto v^{\alpha}$ as follows: $\alpha=-0.77$ for energies $<12.15 \mathrm{eV}$, $\alpha=-1.3$ over the range $12.15 \mathrm{eV} \leqslant h \nu<0.3 \mathrm{keV}, \alpha=-1.6$ for $0.3 \mathrm{keV} \leqslant h v \leqslant 2 \mathrm{keV}$, and $\alpha=-0.8$ for $h v>2 \mathrm{keV}$. We included a low energy cutoff at $1 \mathrm{eV}$ and a high energy cutoff at $100 \mathrm{keV}$. The resulting luminosity in ionizing photons is $Q \approx 2 \times 10^{55}$ photons s $^{-1}$.

The model results are shown in Table 4. The models are deemed to have successfully fit the data when the predicted $\mathrm{H}$ I and either the $\mathrm{C}$ III or the $\mathrm{O}$ VI columns are within one sigma of their measured values. For components 2, 3, and 5, the model over-predicts $\mathrm{O}$ VI, while fitting the $\mathrm{H}$ I and $\mathrm{C} \mathrm{III}$, which suggests that the $\mathrm{O}$ VI is saturated. The one discrepancy for these components is the under-prediction of the $\mathrm{N}$ III in component 2 . To rectify this would require an N/C abundance ratio a factor $\sim 12$ times greater than solar, which seems highly unlikely. The more likely scenario is that the column is due to

\footnotetext{
$5 \quad U=Q / 4 \pi r^{2} c n_{\mathrm{H}}$, where $r$ is the radial distance of the absorber and $Q=\int_{13.6 \mathrm{eV}}^{\infty}\left(L_{v} / h v\right) d v$, or the number of ionizing photons $\mathrm{s}^{-1}$ emitted by a source of luminosity $L_{v}$.
} 
Table 4

CLOUDY Model Parameters and Results ${ }^{\mathrm{a}}$

\begin{tabular}{lcccccc}
\hline \hline Component & $\log N_{H}$ & $\log U_{H}$ & $\mathrm{H} \mathrm{I}^{\mathrm{b}}$ & $\mathrm{O} \mathrm{VI}^{\mathrm{b}}$ & $\mathrm{C} \mathrm{III}^{\mathrm{b}}$ & $\mathrm{N} \mathrm{III}^{\mathrm{b}}$ \\
\hline 1 & 18.6 & -1.15 & $2.3(<9)$ & $8.8(8.9)$ & $0.07(<0.03)$ & $0.01(<0.2)$ \\
2 & 19.7 & -1.30 & $44.3(44.1)$ & $110(>8.8)$ & $1.8(1.8)$ & $0.40(<6.4)$ \\
3 & 19.7 & -1.27 & $40.9(42.3)$ & $112(>8.7)$ & $1.6(1.3)$ & $0.33(<0.6)$ \\
4 & 18.5 & -1.54 & $5.5(5.5)$ & $5.3(5.2)$ & $0.35(<0.5)$ & $0.10(<0.1)$ \\
5 & 20.1 & -1.05 & $59.3(60.1)$ & $288(>14.2)$ & $1.4(1.3)$ & $0.21(<0.6)$ \\
\hline
\end{tabular}

Notes.

${ }^{a}$ Ionic column densities are given in units of $\times 10^{14} \mathrm{~cm}^{-2}$.

${ }^{b}$ Observed values are given in parentheses.

Table 5

O VII Predictions

\begin{tabular}{lc}
\hline \hline Component & $\begin{array}{c}N_{\mathrm{O} \text { VII }} \\
\left(\times 10^{14} \mathrm{~cm}^{2}\right)\end{array}$ \\
\hline 1 & 8.8 \\
2 & 72 \\
3 & 79 \\
4 & 1.9 \\
5 & 361 \\
\hline
\end{tabular}

the $\mathrm{N}$ II ISM contamination in the spectrum. In components 1 and 4, we are able to match the $\mathrm{H}$ I and $\mathrm{O}$ vi columns, indicating that the latter are not saturated. The $\mathrm{C}$ III is over-predicted in the component 1 by a factor of $\sim 2.5$. It is possible that some of the carbon is deposited onto dust grains; there would be no detectable extinction through a cloud with such a low column density, so it is possible that dust is present.

Although Seyferts with intrinsic absorption nearly always possess both UV and X-ray absorption (Crenshaw et al. 2003), the fact that all five components have $\mathrm{O}$ VII column densities $<3 \times 10^{16} \mathrm{~cm}^{-2}$ (Table 5) is consistent with the lack of detectable X-ray absorption in both the ASCA and XMM-Newton spectra of IRAS F22456-5125.

\section{DISTANCE AND DENSITY ESTIMATES}

Assuming that the clouds do remain unchanged between the FUSE observations, which is consistent with these data given the $\mathrm{S} / \mathrm{N}$, we can estimate a lower limit to the distance for these clouds based on the ionization timescale given the increase in flux. If we consider the ionization timescale to be (Krolik \& Kriss 1997)

$$
t_{\text {ion }}=\frac{h \nu}{F_{\text {ion }}\left\langle\sigma_{\text {ion }}\right\rangle}=\frac{h \nu 4 \pi R^{2}}{L_{\text {ion }}\left\langle\sigma_{\text {ion }}\right\rangle},
$$

where $\langle\sigma\rangle_{\text {ion }} \approx 6 \times 10^{-18} \mathrm{~cm}^{2}$ (Osterbrock 1989), $F_{\text {ion }}$ and $L_{\text {ion }}$ are the ionizing flux and luminosity, respectively, and $h v$ is the ionization energy of hydrogen. We calculate an ionizing luminosity from the SED fits from Breeveld et al. (2001) and use the difference in time between the FUSE observations $\left(\Delta t=5.6 \times 10^{7} \mathrm{~s}\right)$ as a lower limit of the ionization timescale $\left(t_{\text {ion }}\right)$ to determine a lower limit to the distance $(R)$. For IRAS F22456-5125, the ionizing luminosity, based on the slopes determined by Breeveld et al. (2001), is $3.0 \times 10^{45} \mathrm{erg} \mathrm{s}^{-1}$. This gives a distance greater than $20 \mathrm{kpc}$ from the central engine.

As we have already demonstrated, several of these components are saturated (i.e., 2, 3, and 5) in O VI and Ly $\beta$. Given that these lines are saturated, we do not expect the observed $\mathrm{O}$ VI and $\operatorname{Ly} \beta$ lines to vary even with substantial changes in column density due to ionization. It is consistent with the large distances, however, that these components do not appear to vary in the higher order Lyman lines, which do not appear to be saturated. The other two components ( 1 and 4$)$ do not appear to be saturated in Ly $\beta$. Thus, we establish the limits on distance for 1 and 4 from $\operatorname{Ly} \beta$, and from the higher order Lyman series for the other three components.

Considering these limits on distance, we can calculate the upper limit of the density for the absorbers via the ionization parameter. For each of the components, we use the equation for ionization parameter and the values from Table 3 to find that the upper limits for the densities are $0.2,0.3,0.3,0.5$, and $0.2 \mathrm{~cm}^{-3}$ for components 1 through 5, respectively.

The distances for several Seyfert galaxy and low-z quasar outflows have been shown to be quite close in, which leads to a more plausible scenario for partial covering of the background source, or an inhomogeneous distribution across the LOS. It is more difficult to explain an absorption cloud located at a large distance from the central source (e.g., $>1 \mathrm{kpc}$ ) with partial covering. While this is difficult, it appears that it is common in outflows as distances of kpc scales for outflows have been seen in several objects thus far. Measurements of excited, metastable state transition lines in broad absorption line (BAL) quasars have shown that several outflows lie at large distances, from $1 \mathrm{kpc}$ to $28 \mathrm{kpc}$ (e.g., Korista et al. 2008; Hamann et al. 2001). In only one of these objects has homogeneous covering been detected (i.e., full covering; Dunn et al. 2010). IRAS F22456-5125 is another object with outflows that likely are located at large distances yet shows partial covering. This could mean that in many AGNs there exists a scattering region significantly larger than the BLR that acts as a background source, which has been supported by spectropolarimetry (e.g., Schmidt \& Hines 1999). Another more physical possibility, given the large distances, is that these outflows are not purely covering a part of the source but that there exists some inhomogeneous distribution of gas across the LOS to the background source that approximates partial covering, as demonstrated by Arav et al. (2005).

\section{SUMMARY AND CONCLUSIONS}

We selected IRAS F22456-5125 out of our sample for further analysis due to its uniqueness. This Seyfert 1 galaxy is a rare object because it shows five distinct narrow lines in O VI, whereas most intrinsic absorbers in $\mathrm{O}$ VI are blended and saturated compared to $\mathrm{C}$ IV and $\mathrm{N}$ v. This allows for measurements of the ionic column densities without contamination from blending.

1. We limit the distance for two of the components from variability to be greater than $20 \mathrm{kpc}$, which likely places this gas in the host galaxy's halo. The other three components are limited in the same fashion, however, due to possible saturation could be interior. 
2. We have shown that, despite the large distances, this gas only partially covers the background source, which is consistent with other absorption systems seen in the literature.

3. Crenshaw et al. (1999) found a 1:1 correlation between UV absorption detection and X-ray warm absorbers in low- $z$ AGNs. We see in the ultrasoft X-ray excess seen by Breeveld et al. (2001) and the excess we find in the $X M M-N e w t o n$ spectrum that there are no signs of X-ray absorption. Furthermore, based on our measurements and Cloudy modeling, we find little evidence for the commonly detected strong O VII X-ray absorption. The lack of X-ray absorption makes IRAS F22456-5125 an exception to the correlation.

4. We note that the SED for IRAS F22456-5125 may have varied as shown by Breeveld et al. (2001). However, in our observations from 2007, we show that the slope is similar to the 1991 observation, with a positively increasing slope to the blue.

As outlined by Hamann et al. (1997), because these outflows exhibit partial covering of the background source they are intrinsically connected with the AGN. However, given the lack of change in the absorption troughs, we must consider that these outflows are located at a large distance from the AGN (on the order of $20 \mathrm{kpc}$ ) and are perhaps not traditional outflows that originated near the accretion disk. The lack of X-ray absorption in this source, unlike all other low- $z$ AGNs, could be a further indication that the outflows are atypical in origin.

However, we cannot rule out that the lack of change in the lines is due to the low $\mathrm{S} / \mathrm{N}$ in the FUSE spectrum. With a higher $\mathrm{S} / \mathrm{N}$ spectra from a high-throughput instrument, such as the Cosmic Origins Spectrograph (COS) on the HST, we could confirm the lack of variability and thus more reliably discuss the nature of the outflow.

This research has made use of the NASA/IPAC Extragalactic Database (NED) which is operated by the Jet Propulsion Laboratory, California Institute of Technology, under contract with the National Aeronautics and Space Administration. This research has also made use of NASA's Astrophysics Data System Abstract Service. We acknowledge support of this research under NASA grants NNG05GC55G, NNG06G185G, and NAG5-13109.

\section{REFERENCES}

Antonucci, R. R. J., \& Miller, J. S. 1985, ApJ, 297, 621

Arav, N., Kaastra, J., Kriss, G. A., Korista, K. T., Gabel, J., \& Proga, D. 2005, ApJ, 620, 665

Breeveld, A. A., Puchnarewicz, E. M., \& Otani, C. 2001, MNRAS, 325, 772

Crenshaw, D. M., Kraemer, S. B., Boggess, A., Maran, S. P., Mushotzky, R. F., \& Wu, C.-C. 1999, ApJ, 516, 750

Crenshaw, D. M., Kraemer, S. B., \& George, I. M. 2003, ARA\&A, 41, 117

Czerny, B., Nikołajuk, M., Różańska, A., Dumont, A.-M., Loska, Z., \& Zycki, P. T. 2003, A\&A, 412, 317

Dixon, W. V. D., Dupuis, J., \& Hurwitz, M. 2002, PASP, 114, 112

Dunn, J. P., Arav, N., Moe, M., Korista, K., Costantini, E., Benn, C., Ellison, S., \& Edmonds, D. 2010, ApJ, 709, 611

Dunn, J. P., Crenshaw, D. M., Kraemer, S. B., \& Gabel, J. R. 2007, AJ, 134, 1061

Dunn, J. P., Crenshaw, D. M., Kraemer, S. B., \& Trippe, M. L. 2008, AJ, 136, 1201

Dunn, J. P., Jackson, B., Deo, R. P., Farrington, C., Das, V., \& Crenshaw, D. M. 2006, PASP, 118, 572

Ferland, G. J., Korista, K. T., Verner, D. A., Ferguson, J. W., Kingdon, J. B., \& Verner, E. M. 1998, PASP, 110, 761

Gebhardt, K., et al. 2003, ApJ, 583, 92

Grevesse, N., \& Anders, E. 1989, in AIP Conf. Ser. 183, Cosmic Abundances of Matter, ed. C. J. Waddington (Melville, NY: AIP), 1

Grupe, D. 2004, AJ, 127, 1799

Grupe, D., Beuermann, K., Thomas, H.-C., Mannheim, K., \& Fink, H. H. 1998, A\&A, 330, 25

Hamann, F. W., Barlow, T. A., Chaffee, F. C., Foltz, C. B., \& Weymann, R. J. 2001, ApJ, 550, 142

Hamann, F., Barlow, T. A., Junkkarinen, V., \& Burbidge, E. M. 1997, ApJ, 478, 80

Khachikian, E. Y., \& Weedman, D. W. 1974, ApJ, 192, 581

Korista, K. T., Bautista, M. A., Arav, N., Moe, M., Costantini, E., \& Benn, C. 2008, ApJ, 688, 108

Kormendy, J., \& Richstone, D. 1995, ARA\&A, 33, 581

Krolik, J. H., \& Kriss, G. A. 1997, in ASP Conf. Ser. 128, Mass Ejection from Active Galactic Nuclei, ed. N. Arav, I. Shlosman, \& R. J. Weymann (San Francisco, CA: ASP), 132

Mason, K. O., et al. 1995, MNRAS, 274, 1194

Osterbrock, D. E. 1989, Astrophysics of Gaseous Nebulae and Active Galactic Nuclei (Research supported by the University of California, John Simon Guggenheim Memorial Foundation, University of Minnesota, et al.; Mill Valley, CA: Univ. Science Books), 422

Piconcelli, E., Jimenez-Bailón, E., Guainazzi, M., Schartel, N., RodríguezPascual, P. M., \& Santos-Lleó, M. 2005, A\&A, 432, 15

Pounds, K. A., et al. 1993, MNRAS, 260, 77

Reeves, J. N., \& Turner, M. J. L. 2000, MNRAS, 316, 234

Savage, B. D., \& Sembach, K. R. 1991, ApJ, 379, 245

Schmidt, G. D., \& Hines, D. C. 1999, ApJ, 512, 125

Seyfert, K. 1943, ApJ, 97, 28

Urry, C. M., \& Padovani, P. 1995, PASP, 107, 803 